\title{
Preoperative three-dimensional versus two-dimensional evaluation in assessment of patients undergoing major liver resection for hepatocellular carcinoma: a propensity score matching study
}

\author{
Pengpeng Li ${ }^{1 \#}$, Mengchao Wang ${ }^{1 \#}$, Yuan Yang ${ }^{1}$, Hui Liu ${ }^{1}$, Zeya Pan ${ }^{1}$, Beige Jiang ${ }^{1}$, Wan Yee Lau ${ }^{2}$, \\ Gang Huang ${ }^{1}$, Weiping Zhou ${ }^{1}$ \\ ${ }^{1}$ Eastern Hepatobiliary Surgery Hospital, Second Military Medical University, Shanghai 200438, China; ${ }^{2}$ Faculty of Medicine, The Chinese \\ University of Hong Kong, Prince of Wales Hospital, Shatin, Hong Kong, China \\ Contributions: (I) Conception and design: WY Lau, W Zhou, G Huang, P Li; (II) Administrative support: None; (III) Provision of study materials \\ or patients: M Wang, Y Yang, H Liu, Z Pan, B Jiang; (IV) Collection and assembly of data: M Wang, B Jiang, P Li, Y Yang; (V) Data analysis and \\ interpretation: W Zhou, P Li, M Wang, G Huang, Z Pan, H Liu; (VI) Manuscript writing: All authors; (VII) Final approval of manuscript: All \\ authors. \\ "These authors contributed equally to this work. \\ Correspondence to: Weiping Zhou, MD; Gang Huang, MD. Eastern Hepatobiliary Surgery Hospital, Second Military Medical University, 225 \\ Changhai Rd., Shanghai 200438, China. Email: ehphwp@126.com; squaror@163.com. Wan Yee Lau, MD, FRCS, FACS, FRACS (Hon). Faculty of \\ Medicine, The Chinese University of Hong Kong, Prince of Wales Hospital, Shatin, Hong Kong, China. Email: josephlau@cuhk.edu.hk.
}

Background: Compared with 2D evaluation, 3D evaluation possesses the virtues of displaying spatial anatomy of intrahepatic blood vessels and its relations to tumors, and enabling calculation of liver volumes, thus facilitating preoperative surgery planning.

Methods: The objective of this study is to study whether preoperative 3D (three-dimensional) evaluation produced better long-term overall survival (OS) outcomes compared to the traditional 2D (two-dimensional) evaluation in patients who underwent major hepatectomy for hepatocellular carcinoma (HCC). This retrospective study matched patients who underwent preoperative $2 \mathrm{D}$ evaluation with those who underwent preoperative $3 \mathrm{D}$ evaluation in a 1:1 ratio using propensity score matching. The primary endpoints were long-term survival outcomes in the two groups after major hepatectomy for HCC.

Results: Of the 248 patients in each of the 2 matched groups, the baseline characteristics were comparable. The median follow-up for all patients was 36 months (range, 0-40 months). The 3-year OS of patients in the PSM cohort was $38.5 \%$. Compared with the 2D Group, patients in the 3D Group had a better OS rate (HR 0.722, 95\% CI: 0.556-0.938, P=0.015) and disease-free survival (DFS) rate (HR 0.741, 95\% CI: 0.590-0.929, $\mathrm{P}=0.009)$. The 3 -year OS and DFS rate for the 3D Group versus the $2 \mathrm{D}$ group were $58.9 \%$ and $44.0 \%$ versus $47.4 \%$ and $33.1 \%$, respectively.

Conclusions: 3D preoperative evaluation resulted in significantly better intermediate-term (3-year) overall survival rate than the traditional $2 \mathrm{D}$ evaluation.

Keywords: Three-dimensional evaluation; major liver resection; hepatocellular carcinoma (HCC); overall survival

Submitted Oct 29, 2019. Accepted for publication Jan 03, 2020.

doi: $10.21037 / \mathrm{atm} .2020 .01 .106$

View this article at: http://dx.doi.org/10.21037/atm.2020.01.106 


\section{Introductions}

Hepatocellular carcinoma (HCC) is the dominating type of liver cancer in many countries, accounting for approximately $80 \%$ of all primary liver cancers (1). Hepatectomy is the most commonly used and effective treatment aiming at cure (2). Accurate preoperative evaluation is of great importance before liver resection to improve safety and effectiveness. For patients with large tumors or with tumors adjacent to major intrahepatic vessels, major liver resections, defined as resecting more than three Couinaud liver segments, have to be performed to eradicate tumors. Twodimensional (2D) evaluation, which is carried out by the 2D images produced by either CT or MRI, has long been used in our institution. The limitations of these 2D images are inadequate exhibition of stereoscopic relationships between major intrahepatic blood vessels and tumors, and inability to precisely calculate the different liver volumes in the planning for operations $(3,4)$. Three-dimensional (3D) evaluation allows these assessments to be done accurately, making this technology rapidly accepted by hepatobiliary surgeons in many institutions (5-8).

Most researches on 3D evaluation focused on its ability to stereoscopically exhibit anatomy, accurately estimate liver volumes, and precisely determine surgical resection margins, thus tremendously help in preoperative surgical planning (9-12). Few studies described the long-term survival outcomes resulted from the benefits obtained in $3 \mathrm{D}$ evaluation. The objective of this study was to determine whether preoperative 3D evaluation produced better longterm overall survival outcomes compared to the traditional preoperative $2 \mathrm{D}$ evaluation.

\section{Methods}

\section{Study design}

A retrospective study was conducted on patients who underwent major liver resections for hepatocellular carcinoma (HCC) and were preoperatively assessed by either $2 \mathrm{D}$ or $3 \mathrm{D}$ evaluation at the Eastern Hepatobiliary Surgery Hospital from January 2012 to December 2018. Patients who underwent preoperative 2D evaluation was matched with patients who underwent preoperative $3 \mathrm{D}$ evaluation in a 1:1 ratio using propensity score matching.

\section{Patients}

The inclusion criteria were: (I) patients with histopathologically confirmed HCC; (II) patients who underwent either preoperative $2 \mathrm{D}$ or $3 \mathrm{D}$ evaluation; (III) Child-Pugh score A and baseline serum alanine aminotransferase (ALT) level $<2$ times the upper limit of normal; and (IV) patients underwent major liver resection defined as resection of more than three Couinaud liver segments.

The exclusion criteria were: (I) patients who had liver tumor other than HCC; (II) patients who had a history of other anti-cancer therapy; (III) patients who had undergone liver resection previously; and (IV) patients had severe dysfunction of heart, lung, kidney or other organs.

\section{Preoperative evaluations}

$2 \mathrm{D}$ evaluation was performed with the traditional $2 \mathrm{D}$ images of CT or MRI. The extent of liver resection and the plane of liver transection were determined by experienced surgeons for radical resection of tumor with a surgical resection margin of at least $1 \mathrm{~cm}$. As far as possible, an anatomical resection based on liver segment/sectors/ hemilivers was done. A non-anatomical wedge resection was only carried out for tumors near important intrahepatic blood vessel which could not be cut off. The patients were not included in this study as these liver resections were not major resections. Vascular resection followed by reconstruction was carried out if clinically indicated.

The $3 \mathrm{D}$-evaluation took advantage of the $3 \mathrm{D}$ visualization model, which has the abilities to 3-dimensionally exhibit intrahepatic anatomy, precisely work out the liver volumes of the future resected specimen and the remnant liver, separate the liver into 8 Couinaud segments, and exactly determine the liver segments where the tumor is located. As a consequence, this model can be used to plan liver resection. Major vascular structures along the planned transection plane can be shown clearly in this model (the Three-dimensional Visualization Software, Shenzhen XuDong Digital Medical Imaging Technology Co., Ltd or the IQQA-Liver EDDA Technology Inc, Princeton, NJ). In the simulation liver resection model, a transection plane was determined by including the tumor with an adequate resection margin in the planned resected specimen. The vascular inflow and outflow to the planned remnant liver were preserved intact. The estimated remnant liver volume by the $3 \mathrm{D}$ visualization model should meet the criteria for liver resection, which was pre-defined as a FLR/SLV ratio $>40 \%(\mathrm{FLR} / \mathrm{BW}>0.8 \%)$ in patients with a background of fibrosis or cirrhosis $(13,14)$, and a ratio $>30 \%$ 
(FLR/BW $>0.5 \%)$ in patients with a normal liver $(15,16)$. If the tumor was close to the middle hepatic vein, extended left/right hepatectomy to include the middle hepatic vein would be performed in patients whose FLR met the predefined requirements.

\section{Operation procedures}

All surgeries were open hepatectomy following the previously reported techniques (17). Intraoperative ultrasound was routinely used to determine the number and location of tumor, and to determine the relationship between tumors and major vessels. The surgery plan would be changed if additional tumors, or severe liver cirrhosis were found during the operations. Liver parenchymal transection was performed using an ultrasonic scalpel (Harmonic Scalpel, HS; Ethicon Endosurgery, USA) or Ligasure (LigaSure, Covidien, USA).

\section{Data collection and follow-up}

Routine preoperatively collected baseline data included intravenous contrast-enhanced CT scan of liver, 3D visualization reconstruction (if carried out) using the Three-dimensional Visualization Software (Shenzhen XuDong Digital Medical Imaging Technology Co., Ltd or the IQQA-Liver EDDA Technology Inc, Princeton, NJ), liver function tests, renal function tests, coagulation profile, complete blood count, chest X-ray, electrocardiogram, lung function tests, gastroscopy, ultrasonic examination of liver and spleen, hepatitis $B$ virus/hepatitis $C$ virus serology tests and tumor markers (AFP, CEA, CA19-9). Echocardiography was carried out in patients older than 60 years.

Intraoperative data collected in this study included operative procedures, duration of operation, time of hepatic inflow occlusion, intraoperative blood loss and volume of blood transfusion. Postoperative data included liver function tests, renal function tests, coagulation profile and complete blood count on postoperative day 1, 3, 5 and 7 , and ultrasonic examination of liver, portal vein, hepatic vein, $\mathrm{X}$-ray chest on postoperative day 4 , postoperative complications (postoperative mortality and morbidity rates) and surgical resection margins. Patients were regularly followed-up in the HCC Clinic. Patients who failed to attend the scheduled follow-up appointments were contacted by a research nurse.

Morbidity was classified according to the Clavien-Dindo classification (18). Minor complications were defined as complication less than grade IIIa. Severe complications were defined as grade IIIb or greater. Post-hepatectomy liver failure (PHLF) was defined and graded using the "ISGLS" criteria (19). Renal failure was defined as an increase of creatinine within 48 hours after surgery to more than 1.4 times of the preoperative level (20). The degrees of liver fibrosis was evaluated using the METAVIR scoring (21). Surgical resection margin was defined as the shortest macroscopic distance from the edge of tumor to the resection plane. $\mathrm{R} 0$ resection was defined as no gross tumor left after resection with non-existence of tumor cells at the plane of transection on microscopic examination.

Patients were followed-up once every month after surgery and re-examined with enhanced CT or MRI, liver function tests, AFP, HBV-DNA and chest radiography once every three months.

The primary endpoint was long-term survival outcomes in the $2 \mathrm{D}$ and $3 \mathrm{D}$ evaluation groups. The secondary endpoints were: (I) Intraoperative data, and (II) postoperative mortality and morbidity rates.

\section{Statistical analysis}

To limit selection bias arising from lack of randomization, Propensity Score Matching (PSM) was performed to match patients who underwent preoperative $2 \mathrm{D}$ evaluation with patients who underwent preoperative $3 \mathrm{D}$ evaluation in a 1:1 ratio using the nearest neighbor matching method with a 0.2 caliper width based on the following variables: age, sex, BMI, ASA score, infection status of HBV, HBVDNA levels, AFP level, platelet count, maximum tumor diameter, tumor number, total bilirubin (TBIL) level, albumin (ALB) level, Alanine transaminase (ALT) level, positivity for microvascular invasion (MVI) and cirrhosis status. Continuous data were expressed as median (range), and categorical data as a count or ratio. The MannWhitney $\mathrm{U}$ test was used as the non-parametric test for two independent samples. Categorical variables were tested by the Pearson's chi-square or Fisher exact tests. The KaplanMeier method was used to estimate overall survival (OS). OS was defined as the time from first treatment to death of all causes. The Log-rank test was used to compare survival outcomes between the two groups. The Cox regressions were carried out to examine the association between OS and demographic and other covariates. All computations relied on the standard software (SPSS Statistics v24; IBM, Chicago, IL). A 2-sided $\mathrm{P}<0.05$ was considered as statistically significant. 
Table 1 Baseline characteristics of patients in the two groups before and after propensity score matching

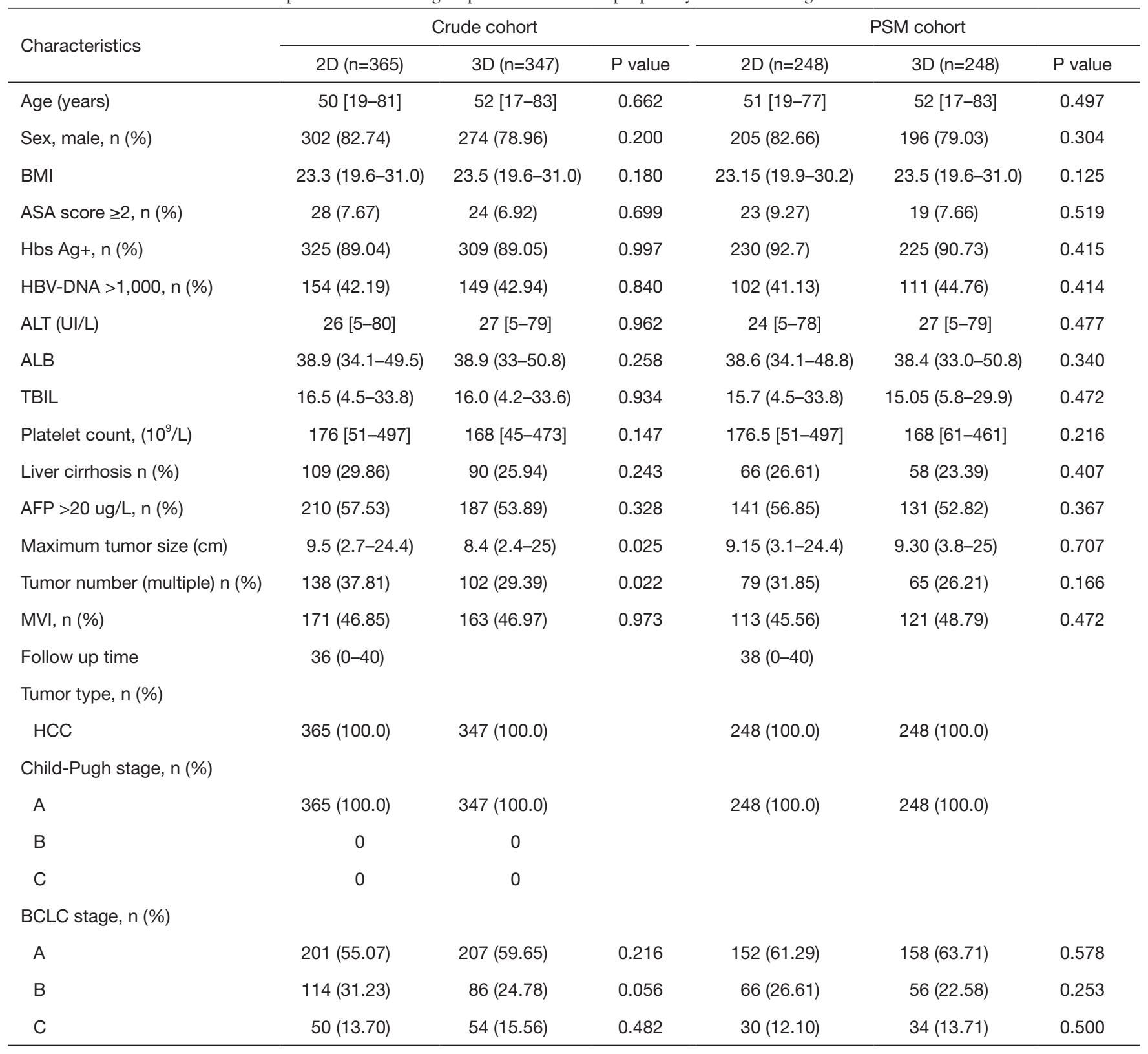

BMI, body mass index; ASA score, The American Society of Anaesthesiologists physical status classification system; TBIL, total bilirubin; ALB, serum albumin; ALT, alanine transaminase; MVI, microvascular invasion; HCC, hepatocellular carcinoma; BCLC stage, Barcelona Clinic Liver Cancer (BCLC) classification.

\section{Results}

From January 2012 to December 2018, 748 patients with HCC underwent major liver resection in our department. Thirty-six patients were excluded because of missing data. Therefore, 712 patients (the 2D group: $n=365$; the $3 \mathrm{D}$ group: $n=347$ ) were eligible for PSM at a 1:1 ratio. After
PSM, 248 patients were matched and included in each of the 2 groups.

\section{Baseline characteristics}

The baseline characteristics of the patients before and after PSM in the 2 groups are shown in Table 1. In the crude 
Table 2 Intraoperative clinical data of patients in the two groups after propensity score matching

\begin{tabular}{|c|c|c|c|}
\hline Characteristics & \multicolumn{2}{|c|}{ Results } & $\mathrm{P}$ values \\
\hline Operating time (min) & $270[180-420]$ & 260 [150-360] & 0.461 \\
\hline Pringle's maneuver, n (\%) & $237(95.6)$ & $229(92.3)$ & 0.123 \\
\hline Pringle's maneuver duration, (min) & $32(0-70)$ & $32(0-60)$ & 0.393 \\
\hline Volume of red blood cells transfusion (units) & $0(0-29)$ & $0(0-16)$ & 0.007 \\
\hline Intraoperative bleeding volume $(\mathrm{mL})$ & $500[200-8,600]$ & $400[200-2,800]$ & 0.018 \\
\hline \multicolumn{4}{|l|}{ Type of hepatectomy, n (\%) } \\
\hline Anatomical resection & $75(30.2)$ & $206(83.1)$ & $<0.001$ \\
\hline
\end{tabular}

cohorts, the $2 \mathrm{D}$ group exhibited a larger maximum tumor diameter ( 9.5 vs. $8.4 \mathrm{~cm}, \mathrm{P}=0.025)$ and a higher proportion of multiple tumors $(37.8 \%$ vs. $29.4 \%, \mathrm{P}=0.022)$ compared with the $3 \mathrm{D}$ group. In the PSM cohorts, there were no significant differences in the baseline characteristics between the two groups.

\section{Intraoperative findings}

The intraoperative data of the two groups of patients after PSM are listed in Table 2. Significant differences in the number of patients who required intraoperative red blood cells transfusion $(\mathrm{P}=0.004)$, and in the volume of intraoperative blood loss $(\mathrm{P}=0.018)$ were found between the two groups. Anatomical liver resection was carried out in 206 of 248 patients $(83.1 \%$ ) in the 3D Group compared with only 75 of 248 patients $(30.2 \%)$ in the 2D Group $(\mathrm{P}<0.001)$. The median resection margin of the $3 \mathrm{D}$ Group was significantly better than the $2 \mathrm{D}$ Group, (median 0.7 , range, $0-1.8 \mathrm{~cm}$ versus median 0.3 , range, $0-1 \mathrm{~cm}$, respectively, $\mathrm{P}<0.001)$.

\section{Postoperative results}

The postoperative complications after PSM are shown in Table 3. Significant differences between the two groups existed in the incidences of postoperative Grade III complications: 30 of 248 patients, or $12.1 \%$ in the $3 \mathrm{D}$ group, and 50 of 248 patients, or $20.1 \%$ in the $2 \mathrm{D}$ group $(\mathrm{P}=0.015)$. The details of the Grade III complications are shown in Table 3. All the postoperative complications responded well to conservative treatment, percutaneous drainage or reoperation. Liver failure ( $\geq$ Grade $\mathrm{C}$ ) happened more frequently but insignificantly in the 2D Group (2.4\%) when compared with the 3D Group (0.4\%) $(\mathrm{P}=0.122)$. Three patients in the 2D Group but no patient in the $3 \mathrm{D}$ group died of PHLF within 90 days of surgery. The causes of 90-day mortality are shown in Table 3.

The median follow-up for all patients was 36 months (range, 0-40 months). And the median follow-up for patients in PSM cohort was 38 months (range, 0-40). The 3-year OS of patients in the crude cohorts was $53.8 \%$. Patients in the 3D Group exhibited better overall survival (OS) rate than patients in the 2D Group (HR $=0.666,95 \% \mathrm{CI}$ : $0.533-0.831, \mathrm{P}<0.001)$. The 3 -year OS rate of the $3 \mathrm{D}$ and 2D groups were $61.0 \%$ and $46.9 \%$, respectively (Figure $1 A$ ). Cox regression analyses showed that evaluation using $3 \mathrm{D}$ (HR $=0.727,95 \%$ CI: 0.578-0.914, $\mathrm{P}=0.006$ ), small tumor $(\mathrm{HR}=1.111,95 \% \mathrm{CI}: 1.081-1.141, \mathrm{P}<0.001)$, solitary tumor $(\mathrm{HR}=1.473,95 \% \mathrm{CI}$ : $1.171-1.853, \mathrm{P}=0.001)$, MVI negativity ( $\mathrm{HR}=1.795,95 \% \mathrm{CI}: 1.416-2.277, \mathrm{P}<0.001$ ), AFP negativity ( $\mathrm{HR}=1.385,95 \% \mathrm{CI}: 1.092-1.757$, $\mathrm{P}=0.007)$ and absence of severe liver fibrosis $(\mathrm{HR}=1.399$, 95\% CI: $1.105-1.772, \mathrm{P}=0.005)$ were significant good risk factors of OS (Table 4). Compared with patients in the 2D Group, patients in the 3D Group also had significantly better disease-free survival (DFS) rate (HR $=0.716,95 \%$ CI: 0.591-0.866, P=0.001) (Figure 1B). The 3-year DFS rate in the $3 \mathrm{D}$ and $2 \mathrm{D}$ groups of patients were $45.1 \%$ versus $32.9 \%$. The 3 -year OS of the patients in the PSM 
Table 3 Postoperative clinical data of patients in the two groups after propensity score matching

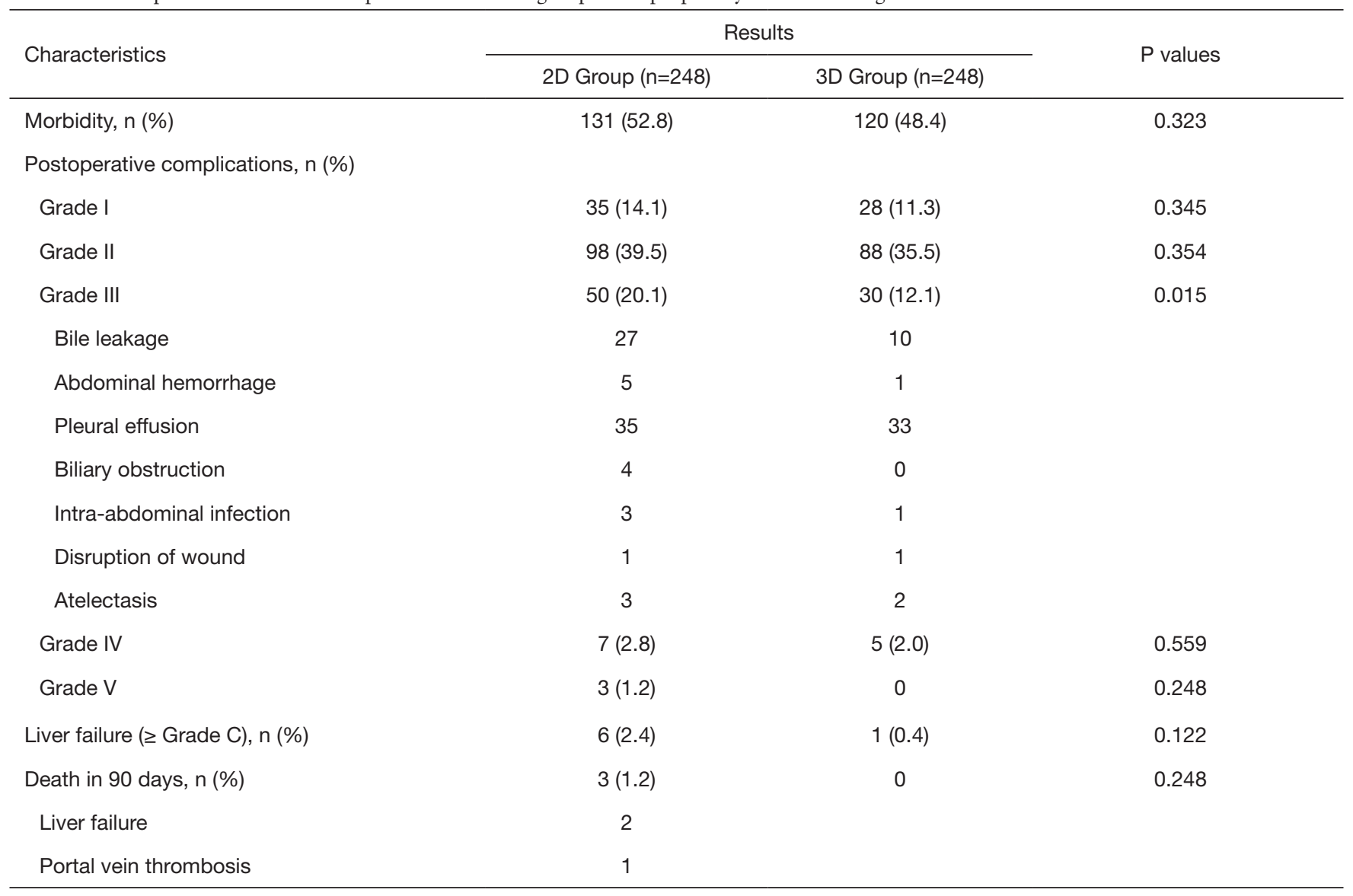

Postoperative complications were classified by Clavin-Dindo classification. Liver failure was defined using the "ISGLS" criteria.
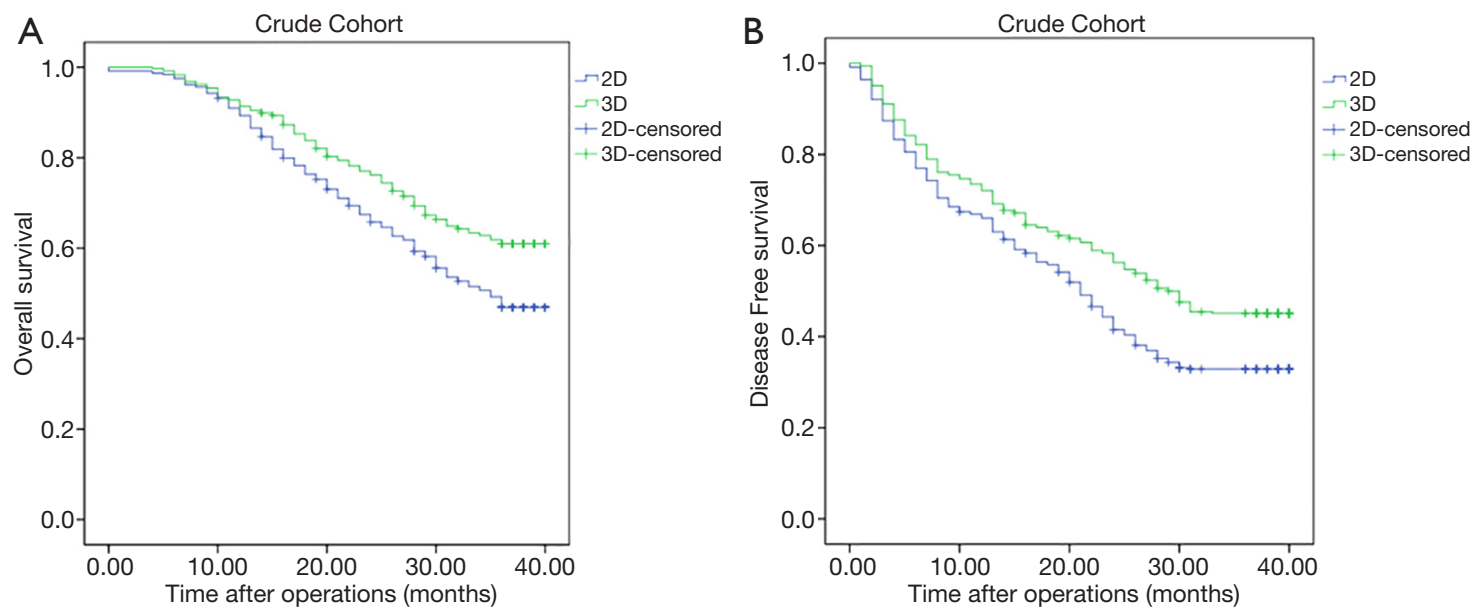

Figure 1 Comparison of the overall survivals (OS) between all the patients in two groups of crude cohorts. Patients in the 3D Group presented with better OS rate than patients in the 2D Group $(\mathrm{P}<0.001)(\mathrm{A})$. Comparison of the disease-free survivals (DFS) between all the patients in two groups of crude cohorts. Patients in the 3D Group also had significantly better DFS rate $(\mathrm{P}=0.001)(\mathrm{B})$. 
Table 4 Uni- and multi-variate analyses for the overall survival results in the crude cohort

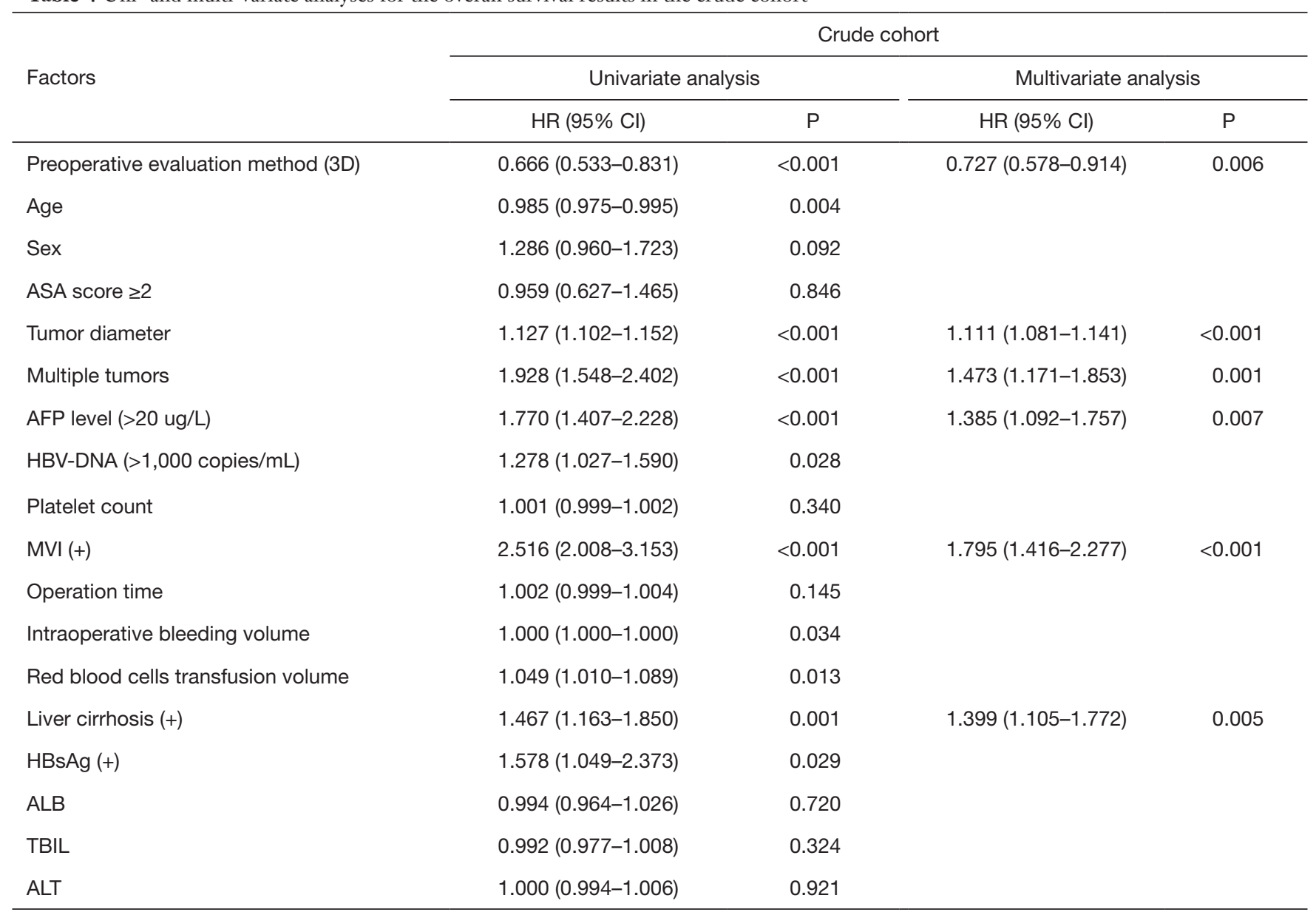

Those variables found significant at $\mathrm{P}<0.2$ in univariable analyses were entered into multivariable Cox-regression analyses.

cohorts was $38.5 \%$. Patients in the 3D Group presented with better overall survival (OS) rate than patients in the 2D Group (HR $=0.722,95 \%$ CI: 0.556-0.938, $\mathrm{P}=0.015$ ). The 3-year OS for all the patients in the two groups was $53.2 \%$. The 3 -year OS rate of the $3 \mathrm{D}$ and $2 \mathrm{D}$ groups were $58.9 \%$ and $47.4 \%$ (Figure $2 A$ ). Cox regression analyses showed that evaluation using $3 \mathrm{D}$ ( $\mathrm{HR}=0.652,95 \% \mathrm{CI}$ : 0.497-0.855, $\mathrm{P}=0.002)$, small tumor ( $\mathrm{HR}=1.084,95 \% \mathrm{CI}$ : $1.047-1.123, \mathrm{P}<0.001)$, solitary tumor $(\mathrm{HR}=2.108,95 \% \mathrm{CI}$ : 1.602-2.773, $\mathrm{P}<0.001$ ), MVI negativity ( $\mathrm{HR}=2.226$, $95 \%$ CI: $1.641-3.020, \mathrm{P}<0.001)$, AFP negativity $(\mathrm{HR}=1.466$, 95\% CI: $1.102-1.951, \mathrm{P}=0.009)$ and absence of severe liver fibrosis (HR $=1.396,95 \%$ CI: $1.041-1.872, \mathrm{P}=0.026$ ) were significant good risk factors of OS (Table 5). Compared with patients in the 2D Group, patients in the 3D Group also had significantly better disease-free survival (DFS) rate (HR $=0.741,95 \%$ CI: $0.590-0.929, \mathrm{P}=0.009$ ) (Figure $2 B$ ). The
3 -year DFS rate of patients was $44.0 \%$ in the 3D Group versus $33.1 \%$ in the $2 \mathrm{D}$ Group.

\section{Discussion}

In our center before August 2014, traditional 2D evaluation was used to evaluate patients before operations and to develop operation plans. In August 2014, the 3D visualization system was introduced into our center. Since then, the number of patients who underwent $3 \mathrm{D}$ evaluation and preoperative planning has gradually been increasing. Now, most complex liver operations are evaluated by $3 \mathrm{D}$ visualization before operations.

Studies have shown that the $3 \mathrm{D}$ visualization technology can facilitate planning and implementation of liver resections, especially in anatomic hepatectomy (6). In our study, $83.1 \%$ of patients in the $3 \mathrm{D}$ evaluation group 

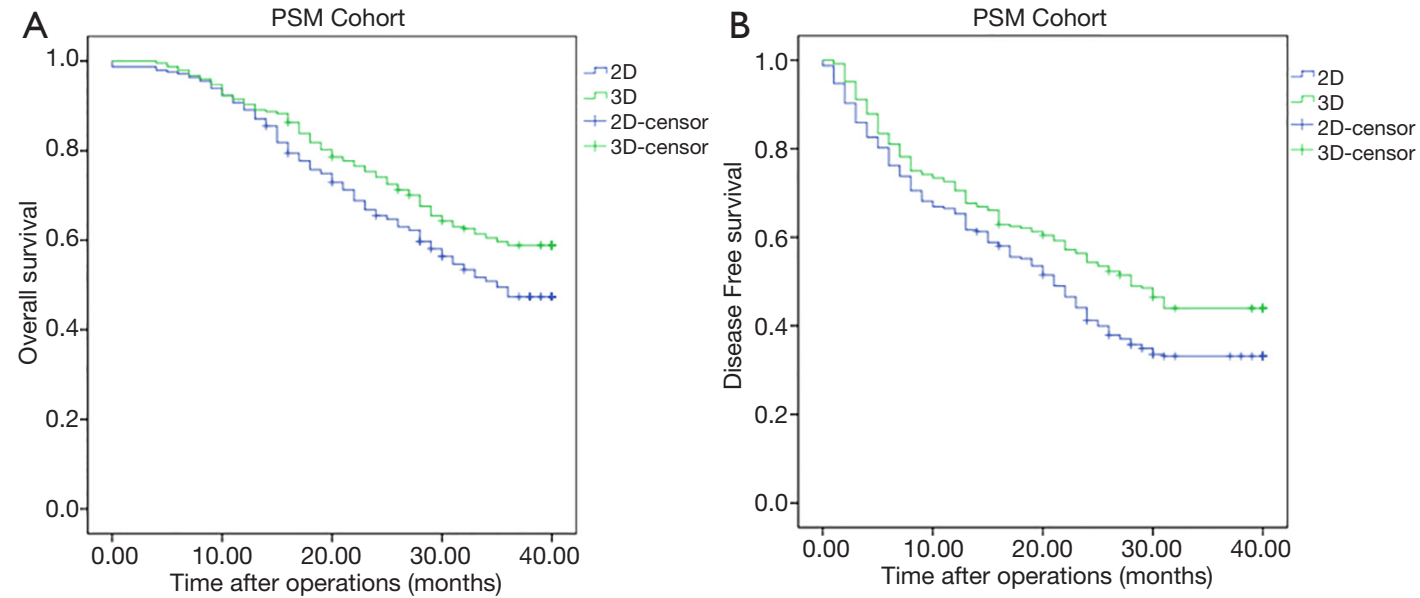

Figure 2 Comparison of the overall survivals (OS) between all the patients in two groups of PSM cohorts. Patients in the 3D Group presented with better OS rate than patients in the 2D Group ( $\mathrm{P}=0.015)(\mathrm{A})$. Comparison of the disease-free survivals (DFS) between all the patients in two groups of PSM cohorts. Patients in the 3D Group also had significantly better DFS rate $(\mathrm{P}=0.009)(\mathrm{B})$.

Table 5 Uni- and multi-variate analyses for the overall survival results in the PSM cohort

\begin{tabular}{|c|c|c|c|c|}
\hline \multirow{3}{*}{ Factors } & \multicolumn{4}{|c|}{ PSM cohort } \\
\hline & \multicolumn{2}{|c|}{ Univariate analysis } & \multicolumn{2}{|c|}{ Multivariate analysis } \\
\hline & $\mathrm{HR}(95 \% \mathrm{Cl})$ & $P$ & $\mathrm{HR}(95 \% \mathrm{Cl})$ & $\mathrm{P}$ \\
\hline Preoperative evaluation method (3D) & $0.722(0.556-0.938)$ & 0.015 & $0.652(0.497-0.855)$ & 0.002 \\
\hline Age & $0.992(0.980-1.003)$ & 0.165 & & \\
\hline ASA score $\geq 2$ & $0.940(0.587-1.503)$ & 0.795 & & \\
\hline Tumor diameter & $1.123(1.093-1.154)$ & $<0.001$ & $1.084(1.047-1.123)$ & $<0.001$ \\
\hline Multiple tumors & $3.108(2.392-4.038)$ & $<0.001$ & 2.108 (1.602-2.773) & $<0.001$ \\
\hline AFP level (>20 ug/L) & $1.848(1.408-2.425)$ & $<0.001$ & $1.466(1.102-1.951)$ & 0.009 \\
\hline $\mathrm{MVI}(+)$ & $3.341(2.536-4.401)$ & $<0.001$ & $2.226(1.641-3.020)$ & $<0.001$ \\
\hline Operation time & $1.002(0.998-1.005)$ & 0.368 & & \\
\hline Intraoperative bleeding volume & $1.000(1.000-1.000)$ & 0.008 & & \\
\hline Red blood cells transfusion volume & $1.076(1.030-1.124)$ & 0.001 & & \\
\hline Liver cirrhosis $(+)$ & $1.544(1.163-2.050)$ & 0.003 & $1.396(1.041-1.872)$ & 0.026 \\
\hline $\mathrm{HBsAg}(+)$ & $3.192(1.576-6.463)$ & 0.001 & & \\
\hline ALB & $0.978(0.937-1.019)$ & 0.288 & & \\
\hline TBIL & $1.000(0.980-1.021)$ & 0.964 & & \\
\hline
\end{tabular}

Those variables found significant at $\mathrm{P}<0.2$ in univariable analyses were entered into multivariable Cox-regression analyses. 
underwent anatomic hepatectomy, which is significantly higher than the $30.2 \%$ of patients in the $2 \mathrm{D}$ evaluation group. There are two possible explanations: (I) preoperative $3 \mathrm{D}$ evaluation can accurately measure the distance between tumors and major intrahepatic vessels, thus providing better planning to the extent of liver resection and the plane of liver transection; (II) by using 3D, the residual liver volumes can be measured accurately to quantitatively assess the risk of postoperative liver failure for the planned operations.

In our study, there were less intraoperative bleeding, less blood transfusion and fewer patients requiring blood transfusion in the $3 \mathrm{D}$ evaluation group. The $3 \mathrm{D}$ visualization model clearly showed the $3 \mathrm{D}$ anatomical structures of the major intrahepatic vessels, anatomic variations, and the relationship between intrahepatic vessels with tumors before the operations. It then enabled surgeons to design a relatively avascular liver plane to transect, to have a good preoperative understanding of which intrahepatic vessels were to be transected, which vessels should be protected and which vessels could be sacrificed during the operations, thus avoiding accidental vascular injuries during operations.

With advances in technology and techniques in liver surgery, and with accumulation of surgical experience, the reported post-hepatectomy morbidity $(47.7 \%)$ and mortality $(4.7 \%)$ rates are still high $(22,23)$. Liver failure is the most dreaded post-hepatectomy complication and the leading cause of death $(19,24-26)$. The main causes of post-hepatectomy liver failure in the $2 \mathrm{D}$ imaging era are the difficulties in preoperative identification of intrahepatic vascular anomalies, and the accurate assessment of the volume of future liver remnant. Injury of intrahepatic vascular anomalies during operation can lead to ischemic necrosis or congestion of liver parenchyma, causing postoperative liver failure. Many studies have shown a significant correlation between the volume of future liver remnant with postoperative complications (27-29). The 3D visualization technology can accurately assess the extent of hepatectomy before operation, preserve adequate residual liver volumes, avoid damage to important intrahepatic vessels, ensure complete blood inflow and outflow to liver remnants, thus significantly reducing the chance of hepatic parenchymal necrosis, ischemia or congestion after hepatectomy, and the incidences of liver failure and death due to inadequate residual liver volume. The preoperative $3 \mathrm{D}$ visualization assessment leads to more anatomic resection and helps surgeons to design more reasonable liver transection and relatively less vascular planes to go through. All these contribute to less intraoperative bleeding and postoperative bile leak. As a consequence, the incidences of major complications (> Grade III) and posthepatectomy hepatic failure for patients in the 3D Group were significantly lower than the 2D Group. Three patients died within 90 days after surgery in the 2D Group, while there were no deaths in the 3D Group. With heterogeneous distribution of liver function in livers (30,31), using liver volumetry alone is not enough to assess liver functional reserve of patients. Functional assessment is effective in supplementing liver volume evaluation (32). The (99m) Tc-GSA scintigraphy SPECT-CT fusion system can estimate correct functional liver volume and it is useful in comparison with conventional CT volumetry (33).

Anatomic hepatectomy with adequate resection margins are important factors relating to better long-term survival outcomes of patients (34-38). Our study supported that 3D evaluation was better than the traditional $2 \mathrm{D}$ evaluation in the 3 -year overall survival rate of patients.

This study has several limitations. First, although this study used PSM to minimize the unbalanced baseline characteristics of patients in the two study groups, inherent biases can still occur in this retrospective study. Second, while most of the 2D group of patients were treated from January 2012 to August 2014, most of the 3D group of patients were treated from August 2014 to December 2018. The increase in experience in liver resection can explain at least partly the better treatment outcomes in the $3 \mathrm{D}$ evaluation group. Third, some preoperative examinations could not even be performed in the two groups, e.g., ICG-R15 and Fibro-scan. This can result in our inability to use some accurate indicators to assess the state of liver function and fibrosis in selecting variables to generate the propensity score model. Fourth, in this study, only patients with hepatocellular carcinoma who underwent major liver resections were included. Therefore, the significance of preoperative $3 \mathrm{D}$ evaluation on patients with less extensive hepatectomy was not studied.

\section{Acknowledgments}

The authors thank the staff of the Medical Image Center, Eastern Hepatobiliary Surgery Hospital for help during the study.

Funding: This study was funded by the Science Fund for Creative Research Groups, NSFC, China (81521091); the State Key Infection Disease Project of China (2018ZX10732202002-005) and the National Human Genetic Resources Sharing 
Service Platform (2005DKA21300).

\section{Footnote}

Conflicts of Interest: The authors have no conflicts of interest to declare.

Ethical Statement: The authors are accountable for all aspects of the work in ensuring that questions related to the accuracy or integrity of any part of the work are appropriately investigated and resolved. The study was approved by the Ethics Committee of the Eastern Hepatobiliary Surgery Hospital.

Open Access Statement: This is an Open Access article distributed in accordance with the Creative Commons Attribution-NonCommercial-NoDerivs 4.0 International License (CC BY-NC-ND 4.0), which permits the noncommercial replication and distribution of the article with the strict proviso that no changes or edits are made and the original work is properly cited (including links to both the formal publication through the relevant DOI and the license). See: https://creativecommons.org/licenses/by-nc-nd/4.0/.

\section{References}

1. Ozakyol A. Global Epidemiology of Hepatocellular Carcinoma (HCC Epidemiology). J Gastrointest Cancer 2017;48:238-40.

2. Kawaguchi Y, Honda G, Endo I, et al. Current Technical Issues for Surgery of Primary Liver Cancer. Liver Cancer 2016;6:51-8.

3. Wei XB, Xu J, Li N, et al. The role of three-dimensional imaging in optimizing diagnosis, classification and surgical treatment of hepatocellular carcinoma with portal vein tumor thrombus. HPB 2016;18:287-95.

4. Fang CH, Tao H, Yang J, et al. Impact of ThreeDimensional Reconstruction Technique in the Operation Planning of Centrally Located Hepatocellular Carcinoma. J Am Coll Surg 2015;220:28-37.

5. Hallet J, Gayet B, Tsung A, et al., Systematic review of the use of pre-operative simulation and navigation for hepatectomy: current status and future perspectives. J Hepatobiliary Pancreat Sci 2015;22:353-62.

6. Takamoto T, Hashimoto T, Ogata S, et al. Planning of anatomical liver segmentectomy and subsegmentectomy with 3-dimensional simulation software. Am J Surg 2013;206:530-8.
7. Oshiro Y, Ohkohchi N. Three-Dimensional Liver Surgery Simulation: Computer-Assisted Surgical Planning with Three-Dimensional Simulation Software and ThreeDimensional Printing. Tissue Eng Part A 2017;23:474-80.

8. Mise Y, Hasegawa K, Satou S, et al. How Has Virtual Hepatectomy Changed the Practice of Liver Surgery?: Experience of 1194 Virtual Hepatectomy Before Liver Resection and Living Donor Liver Transplantation. Ann Surg 2018;268:127-33.

9. Wigmore SJ, Redhead DN, Yan XJ, et al. Virtual Hepatic Resection Using Three-Dimensional Reconstruction of Helical Computed Tomography Angioportograms. Ann Surg 2001;233:221-6.

10. Saito S, Yamanaka J, Miura K, et al. A novel 3D hepatectomy simulation based on liver circulation: application to liver resection and transplantation. Hepatology 2005;41:1297-304.

11. Yamanaka J, Saito S, Fujimoto J. Impact of preoperative planning using virtual segmental volumetry on liver resection for hepatocellular carcinoma. World J Surg 2007;31:1249-55.

12. Radtke A, Sotiropoulos GC, Molmenti EP, et al. Computer-Assisted Surgery Planning for Complex Liver Resections: When Is It Helpful? A SingleCenter Experience Over an 8-Year Period. Ann Surg 2010;252:876-83.

13. Kiuchi T, Kasahara M, Uryuhara K, et al. Impact of graft size mismatching on graft prognosis in liver transplantation from living donors. Transplantation 1999;67:321-7.

14. Abdalla EK, Adam R, Bilchik AJ, et al. Improving Resectability of Hepatic Colorectal Metastases: Expert Consensus Statement. Ann Surg Oncol 2006;13:1271-80.

15. Truant S, Oberlin O, Sergent G, et al. Remnant Liver Volume to Body Weight Ratio $\geq 0.5 \%$ : A New Cut-Off to Estimate Postoperative Risks after Extended Resection in Noncirrhotic Liver. J Am Coll Surg 2007;204:22-33.

16. Yigitler C, Farges O, Kianmanesh R, et al. The small remnant liver after major liver resection: How common and how relevant? Liver Transpl 2003;9:S18-25.

17. Yang Y, Lai ECH, Fu SY, et al. A prospective randomized controlled trial to compare two methods of selective hepatic vascular exclusion in partial hepatectomy. Eur J Surg Oncol 2013;39:125-30.

18. Dindo D, Demartines N, Clavien PA. Classification of Surgical Complications: A New Proposal With Evaluation in a Cohort of 6336 Patients and Results of a Survey. Ann Surg 2004;240:205-13.

19. Rahbari NN, Garden OJ, Padbury R, et al. 
Posthepatectomy liver failure: a definition and grading by the International Study Group of Liver Surgery (ISGLS). Surgery 2011;149:713-24.

20. Lameire N, Biesen WV, Vanholder R. Acute kidney injury. Lancet 2008;372:1863-5.

21. Bedossa P, Poynard T. An algorithm for the grading of activity in chronic hepatitis C. Hepatology 1996;24:289-93.

22. Mullen JT, Ribero D, Reddy SK, et al. Hepatic insufficiency and mortality in 1,059 noncirrhotic patients undergoing major hepatectomy. J Am Coll Surg 2007;204:854-62; discussion 862-4.

23. Jin S. Management of post-hepatectomy complications. World J Gastroenterol 2013;19:7983.

24. Holt DR, Thiel DV, Edelstein S, et al. Hepatic resections. Arch Surg Chic Ill 1960 2000;135:1353-8.

25. van den Broek MAJ, Olde Damink SWM, Dejong CHC, et al. Liver failure after partial hepatic resection: definition, pathophysiology, risk factors and treatment. Liver Int 2008;28:767-80.

26. Schreckenbach T, Liese J, Bechstein WO, et al. Posthepatectomy liver failure. Dig Surg 2012;29:79-85.

27. Schindl MJ. The value of residual liver volume as a predictor of hepatic dysfunction and infection after major liver resection. Gut 2005;54:289-96.

28. Ribero D, Abdalla EK, Madoff DC, et al. Portal vein embolization before major hepatectomy and its effects on regeneration, resectability and outcome. Br J Surg 2007;94:1386-94.

29. Kishi Y, Abdalla EK, Chun YS, et al. Three hundred and one consecutive extended right hepatectomies: evaluation of outcome based on systematic liver volumetry. Ann Surg 2009;250:540-8.

30. de Graaf W, van Lienden KP, van Gulik TM, et al. 99mTc-Mebrofenin Hepatobiliary Scintigraphy with SPECT for the Assessment of Hepatic Function and Liver
Functional Volume Before Partial Hepatectomy. J Nucl Med 2010;51:229-36.

31. Nilsson H, Karlgren S, Blomqvist L, et al. The inhomogeneous distribution of liver function: possible impact on the prediction of post-operative remnant liver function. HPB 2015;17:272-7.

32. Kwon AH, Matsui Y, Ha-Kawa SK, et al. Functional hepatic volume measured by technetium-99m-galactosylhuman serum albumin liver scintigraphy: comparison between hepatocyte volume and liver volume by computed tomography. Am J Gastroenterol 2001;96:541-6.

33. Beppu T, Hayashi H, Okabe H, et al. Liver functional volumetry for portal vein embolization using a newly developed 99mTc-galactosyl human serum albumin scintigraphy SPECT-computed tomography fusion system. J Gastroenterol 2011;46:938-43.

34. Ikai I, Arii S, Kojiro M, et al. Reevaluation of prognostic factors for survival after liver resection in patients with hepatocellular carcinoma in a Japanese nationwide survey. Cancer 2004;101:796-802.

35. Shimada K, Sakamoto Y, Esaki M, et al. Role of the width of the surgical margin in a hepatectomy for small hepatocellular carcinomas eligible for percutaneous local ablative therapy. Am J Surg 2008;195:775-81.

36. Regimbeau JM, Kianmanesh R, Farges O, et al. Extent of liver resection influences the outcome in patients with cirrhosis and small hepatocellular carcinoma. Surgery 2002;131:311-7.

37. Hasegawa K, Kokudo N, Imamura H, et al. Prognostic Impact of Anatomic Resection for Hepatocellular Carcinoma. Ann Surg 2005;242:252-9.

38. Ishii M, Mizuguchi T, Kawamoto M, et al. Propensity score analysis demonstrated the prognostic advantage of anatomical liver resection in hepatocellular carcinoma. World J Gastroenterol 2014;20:3335-42.
Cite this article as: Li P, Wang M, Yang Y, Liu H, Pan Z, Jiang B, Lau WY, Huang G, Zhou W. Preoperative threedimensional versus two-dimensional evaluation in assessment of patients undergoing major liver resection for hepatocellular carcinoma: a propensity score matching study. Ann Transl Med 2020;8(5):182. doi: 10.21037/atm.2020.01.106 